\title{
Life Analysis and Reliability Prediction of Micro-Switches based on Life Prediction Method
}

\author{
Jung-Geon Ji*, Kun-Young Shin*, Duk-Gyu Lee*, Moon-Shuk Song** and Hi Sung Lee ${ }^{\dagger}$
}

\begin{abstract}
Reliability means that a product maintains its initial quality and performance at a certain period of time (time, distance, cycle etc) under given condition without failure. The given conditions include both environmental condition and operating condition. Environmental condition means a common natural environment such as temperature, humidity, vibration, and working condition means an artificial environment such as voltage, current load, place for installment, and hours of use, which occurs during the life of the product. In the field of railway vehicles, it is mandatory to use a part with the proved reliability as the extension of the life of vehicle become highly necessary. But the reliable assessment method for the reliability of the part is insufficient. If the reliability of the railway vehicle parts could be assessed by using the field data, the reliability of the entire system could also be evaluated reliably. In this study, life span of micro-switch for master controller is analyzed and prediction is performed based on its field data given by an operator considering the special circumstances of railway vehicles such as the operation of a large number of trains on the same line.
\end{abstract}

Key words : Reliability prediction, Life analysis, Micro-switches, Life prediction method

\section{Introduction}

Reliability means that a product maintains its initial quality and performance at certain period of time (time, distance, cycle etc) under given condition without failures. Given conditions include both environmental condition and operating condition. Environmental condition means common natural environment such as temperature, humidity, vibration, and working condition means artificial environment such as voltage, current load, installation place, and hours of use, which occurs during using the product.

In this study, life span of micro-switch for master controller is analyzed and prediction is performed based on its field data collected at metro line number A, considering the special circumstances of railway operator, such as the operation of a large number of trains on the same line where parts are often worn out and deteriorated by heat.

\footnotetext{
$\dagger$ Corresponding author: Professor, Graduate School of Railroad, Seoul National University of Science \& Technology

E-mail : hslee@seoultech.ac.kr

* Seoul Metropolitan Rapid Transit Corporation

${ }^{* *}$ Korea National University of Transportation
}

\section{Prediction Method of Reliability and Life Span}

Reliability prediction is to predict failure rate and average life span associated with reliability by taking operation, working environment and conditions associated with parts and systems into account. Table 1 refers to types and measures of life span prediction. By conducting reliability prediction, manufacturers can predict product life span and consumers can have a choice for product with longer life span. In terms of railway vehicles, many prediction standards allow us to predict failure rates of parts under a given system operation and working condition. The prediction will eventually be used in design and product development stage of parts and system. Also the prediction enables the operators to secure customer safety by building preventive maintenance plan in advance.

Predicting the life span of parts and applying it to maintenance or conducting activities to promote longer life span by reflecting operation data feedback to a design are not yet a common practice in railway vehicles area and it is quite challenging. Operator is conduction maintenance 
Table 1. Types of Life Span Prediction and Its Method

\begin{tabular}{c|c|c|c}
\hline No. & Types of life span prediction & Prediction method & Comment \\
\hline 1 & $\begin{array}{c}\text { Prediction with a reference value given by } \\
\text { manufacturer }\end{array}$ & $\begin{array}{c}\text { Prediction with maintenance standards } \\
\text { given by manufacturer }\end{array}$ & $\begin{array}{c}\text { Modified the result at cycle stage test or lab } \\
\text { data is being used. Data differ from actual } \\
\text { environment }\end{array}$ \\
\hline 3 & $\begin{array}{c}\text { Prediction considering operation } \\
\text { characteristics }\end{array}$ & $\begin{array}{c}\text { Set reference value by considering } \\
\text { operation characteristics and predict life } \\
\text { span }\end{array}$ & $\begin{array}{c}\text { Lack of adaptability towards operation } \\
\text { environment change and require longer } \\
\text { time period. }\end{array}$ \\
\hline 4 & Prediction by using field data & $\begin{array}{c}\text { Statistical life span prediction by using field } \\
\text { data on defect occurrence history }\end{array}$ & $\begin{array}{c}\text { Secured field data with quality that meets } \\
\text { statistically needed information(usage time, } \\
\text { failure type) }\end{array}$ \\
\hline 5 & Prediction by conducting an accelerated life & $\begin{array}{c}\text { Conduct test under severe conditions until } \\
\text { failure takes place and then use a model to } \\
\text { calculate life under a normal condition. }\end{array}$ & $\begin{array}{c}\text { Types of stresses variable by parts which } \\
\text { consist the product }\end{array}$ \\
\hline
\end{tabular}

Table 2. Natural Life Data (by manufacturer) by Relay

\begin{tabular}{c|c|c}
\hline Category & No. 5 line & No. 6,7,8 lines \\
\hline Manufacturer & HONEYWELL & SCHALTBAU \\
\hline $\begin{array}{c}\text { Photo of the } \\
\text { parts }\end{array}$ & \\
\hline & Electrical life: & Electrical life : \\
Life span & Not disclosed & 1 Million times \\
& Mechanical life : & Mechanical life : \\
& 10 Million times & 10 Million times \\
\hline
\end{tabular}

activities set forth in Table 1 which is given by manufacturers. However, the manufacturers do not provide maintenance guidelines which consider life span or parts characteristics except the cases of simple consumables such as oil and rubber. Life span evaluation can be divided into 5 big categories. 1, 2, 3 can be adopted by operator as a prediction measures and 4, 5 can be adopted by manufacturer as factors to be considered at development stage. $^{[1]}$

\section{Prediction with a Reference Value given by Manufacturer}

Micro-switch manufacturer presents natural life by referring operating frequency, not by MTBF and a failure rate. Presented values are the same as the data in Table 2, and it is consisted with mechanical life decided by physical stress and electrical life decided by contacts' current
Table 3. Usage Frequency Level Based on Operating Frequency

\begin{tabular}{|c|c|c|c|c|c|}
\hline $\begin{array}{l}\text { Usage } \\
\text { level }\end{array}$ & Items & $\begin{array}{l}\text { Operating } \\
\text { frequency }\end{array}$ & $\begin{array}{l}\text { Annual } \\
\text { running } \\
\text { distance }\end{array}$ & $\begin{array}{l}\text { Operation } \\
\text { distance and } \\
\text { number of } \\
\text { stations }\end{array}$ & $\begin{array}{l}\text { Annual } \\
\text { average } \\
\text { round trips }\end{array}$ \\
\hline \multirow{2}{*}{6} & P1 & 332 & \multirow{17}{*}{$114,773 \mathrm{~km}$} & \multirow{17}{*}{$46.9 \mathrm{~km} / 42$} & \multirow{17}{*}{$\begin{array}{l}1,224 \\
\text { times }\end{array}$} \\
\hline & $\mathrm{P} 2$ & 332 & & & \\
\hline \multirow{4}{*}{5} & P8 & 176 & & & \\
\hline & P9 & 176 & & & \\
\hline & P5 & 175 & & & \\
\hline & P6 & 175 & & & \\
\hline \multirow{2}{*}{4} & P3 & 156 & & & \\
\hline & P4 & 156 & & & \\
\hline 3 & DMS & 146 & & & \\
\hline \multirow{4}{*}{2} & D1 & 2 & & & \\
\hline & D2 & 2 & & & \\
\hline & D3 & 2 & & & \\
\hline & D8 & 2 & & & \\
\hline \multirow{4}{*}{1} & D4 & - & & & \\
\hline & D6 & - & & & \\
\hline & D7 & - & & & \\
\hline & P7 & - & & & \\
\hline
\end{tabular}

conduction, voltage and breaking current. As microswitch operating rate is different under different working environment and purpose, simple operating frequency number is not sufficient to be reference standards for manufactures. 
Table 4. Micro-switch FMEA

\begin{tabular}{|c|c|c|c|c|c|c|c|c|c|c|}
\hline Items & Level & Description & $\begin{array}{l}\text { Failure } \\
\text { Mode }\end{array}$ & $\begin{array}{l}\text { Failure } \\
\text { Cause }\end{array}$ & $\begin{array}{l}\text { Local } \\
\text { Effects }\end{array}$ & $\begin{array}{l}\text { Final } \\
\text { Effects }\end{array}$ & $\begin{array}{l}\text { Failure } \\
\text { Detection }\end{array}$ & Severity & $\begin{array}{l}\text { Occurrence } \\
\text { Frequency }\end{array}$ & $\begin{array}{c}\text { Failure } \\
\text { effects } \\
\text { Probability }\end{array}$ \\
\hline $\begin{array}{l}\text { Direction } \\
\text { Controller }\end{array}$ & 2 & $\begin{array}{c}\text { Direction } \\
\text { authorization }\end{array}$ & FSB failure & $\begin{array}{c}\text { Tension } \\
\text { failure of } \\
\text { Handle } \\
\text { spring }\end{array}$ & $\begin{array}{c}\text { Recognition } \\
\text { failure of } \\
\mathrm{P}, \mathrm{B}, \mathrm{N} \\
\text { authorization }\end{array}$ & $\begin{array}{l}\text { Change of } \\
\text { train } \\
\text { operation }\end{array}$ & $\begin{array}{c}\text { Operation } \\
\text { and function } \\
\text { check }\end{array}$ & 1 & 1 & 1 \\
\hline Camswitch & 3 & $\begin{array}{l}\text { Signal transfer to } \\
\text { microswitch }\end{array}$ & $\begin{array}{c}\text { FSB } \\
\text { failure }\end{array}$ & \begin{tabular}{|c|} 
Cam \\
Deformation
\end{tabular} & ditto & ditto & ditto & 1 & 1 & 1 \\
\hline D1 Micro-switch & 4 & $\begin{array}{c}\text { Direction } \\
\text { controller forward } \\
\text { TC recognition }\end{array}$ & $\begin{array}{c}\mathrm{TE} / \mathrm{BE} \\
\text { fault(711),P, } \\
\mathrm{B}, \mathrm{N} \\
\text { recognition } \\
\text { fault(611) }\end{array}$ & \begin{tabular}{|c|} 
Contact \\
failure of \\
microswitch
\end{tabular} & ditto & ditto & $\begin{array}{c}\text { Measuremen } \\
t \text { of contact } \\
\text { resistance }\end{array}$ & 3 & 2 & 1 \\
\hline D2 Micro-switch & 4 & ditto & ditto & ditto & ditto & ditto & ditto & 3 & 2 & 1 \\
\hline D3 Micro-switch & 4 & ditto & ditto & ditto & ditto & ditto & ditto & 3 & 2 & 1 \\
\hline D4 Micro-switch & 4 & ditto & ditto & ditto & ditto & ditto & ditto & 1 & 1 & 1 \\
\hline D5 Micro-switch & 4 & ditto & ditto & ditto & ditto & ditto & ditto & 1 & 1 & 1 \\
\hline D6 Micros-witch & 4 & ditto & ditto & ditto & ditto & ditto & ditto & 1 & 1 & 1 \\
\hline D7 Micro-switch & 4 & ditto & ditto & ditto & ditto & ditto & ditto & 1 & 1 & 1 \\
\hline D8 Micro-switch & 4 & $\begin{array}{r}\text { when Forward and } \\
\text { Reverse, keeping } \\
\text { battery contactor } \\
\end{array}$ & ditto & ditto & ditto & ditto & ditto & 1 & 2 & 1 \\
\hline $\begin{array}{l}\text { Powering } \\
\text { Controller }\end{array}$ & 2 & $\begin{array}{l}\text { powering/braking } \\
\text { handling }\end{array}$ & FSB failure & $\begin{array}{l}\text { Tension } \\
\text { failure of } \\
\text { Handle } \\
\text { spring }\end{array}$ & ditto & ditto & $\begin{array}{c}\text { Operation } \\
\text { and function } \\
\text { check }\end{array}$ & 1 & 1 & 1 \\
\hline Cam-switch & 3 & $\begin{array}{l}\text { Signal transfer to } \\
\text { microswitch }\end{array}$ & ditto & $\begin{array}{c}\text { Cam } \\
\text { Deformation }\end{array}$ & ditto & ditto & \begin{tabular}{|c|}
$\begin{array}{c}\text { Operation } \\
\text { and function } \\
\text { check }\end{array}$ \\
\end{tabular} & 1 & 1 & 1 \\
\hline P1 Micro-switch & 4 & \begin{tabular}{|c|} 
B7 $\sim \mathrm{P} 4$ recognition \\
of TC and $\mathrm{CC} 1$
\end{tabular} & $\begin{array}{c}\mathrm{TE} / \mathrm{BE} \\
\text { fault(711),P, } \\
\mathrm{B}, \mathrm{N} \\
\text { recognition } \\
\text { fault(611) }\end{array}$ & \begin{tabular}{|c|} 
Contact \\
failure of \\
microswitch
\end{tabular} & ditto & ditto & $\left|\begin{array}{c}\text { Measuremen } \\
t \text { of contact } \\
\text { resistance }\end{array}\right|$ & 5 & 6 & 1 \\
\hline P2 Micro-switch & 4 & $\begin{array}{c}\text { B7 P4 recognition } \\
\text { (emergency } \\
\text { braking safetu } \\
\text { loop) }\end{array}$ & ditto & ditto & ditto & ditto & ditto & 5 & 6 & 1 \\
\hline P3 Micro-switch & 4 & $\begin{array}{l}\text { B7 } \sim \mathrm{B} 1 \text { braking } \\
\text { recognition }\end{array}$ & ditto & ditto & ditto & ditto & ditto & 4 & 4 & 1 \\
\hline P4 Microswitch & 4 & $\begin{array}{l}\text { B7 B1 braking } \\
\text { recognition } \\
\text { (emergency } \\
\text { powering) }\end{array}$ & ditto & ditto & ditto & ditto & ditto & 4 & 4 & 1 \\
\hline P5 Microswitch & 4 & $\begin{array}{c}\text { Neutral } \\
\text { recognition } \\
(\mathrm{TCMS})\end{array}$ & ditto & ditto & ditto & ditto & ditto & 4 & 5 & 1 \\
\hline P6 Microswitch & 4 & $\begin{array}{l}\text { Neutral } \\
\text { recognition } \\
\text { (ATC) }\end{array}$ & ditto & ditto & ditto & ditto & ditto & 2 & 5 & 1 \\
\hline
\end{tabular}


Table 4. Continued

\begin{tabular}{|c|c|c|c|c|c|c|c|c|c|c|}
\hline Items & Level & Description & $\begin{array}{l}\text { Failure } \\
\text { Mode }\end{array}$ & $\begin{array}{l}\text { Failure } \\
\text { Cause }\end{array}$ & $\begin{array}{l}\text { Local } \\
\text { Effects }\end{array}$ & $\begin{array}{l}\text { Final } \\
\text { Effects }\end{array}$ & $\begin{array}{l}\text { Failure } \\
\text { Detection }\end{array}$ & Severity & $\begin{array}{l}\text { Occurrence } \\
\text { Frequency }\end{array}$ & $\begin{array}{c}\text { Failure } \\
\text { effects } \\
\text { Probability }\end{array}$ \\
\hline P7 Microswitch & 4 & $\begin{array}{l}\text { Full service brake } \\
\text { recognition(ATC) }\end{array}$ & ditto & ditto & ditto & ditto & ditto & 2 & 1 & 1 \\
\hline P8 Microswitch & 4 & $\begin{array}{c}\text { P0.5 P4 powering } \\
\text { recognition(TCM } \\
\mathrm{S})\end{array}$ & ditto & ditto & ditto & ditto & ditto & 3 & 5 & 1 \\
\hline P9 Microswitch & 4 & $\begin{array}{c}\text { P0.5 P4 powering } \\
\text { recognition(emerg } \\
\text { ency powering) }\end{array}$ & ditto & ditto & ditto & ditto & ditto & 3 & 5 & 1 \\
\hline Deadman Handle & 2 & Deadman handle & ditto & $\begin{array}{l}\text { Tension } \\
\text { failure of } \\
\text { Handle } \\
\text { spring }\end{array}$ & ditto & ditto & ditto & 1 & 1 & 1 \\
\hline $\begin{array}{c}\text { DMS1 } \\
\text { Microswitch }\end{array}$ & 3 & $\begin{array}{l}\text { Deadman signal } \\
\text { recognition }\end{array}$ & ditto & $\begin{array}{c}\text { Contact } \\
\text { failure of } \\
\text { microswitch }\end{array}$ & ditto & ditto & ditto & 4 & 3 & 1 \\
\hline $\begin{array}{c}\text { DMS2 } \\
\text { Microswitch }\end{array}$ & 3 & ditto & ditto & ditto & ditto & ditto & ditto & 4 & 3 & 1 \\
\hline
\end{tabular}

\section{Prediction Considering Operation Characteristics}

\subsection{Operating frequency analysis based on operation distance}

Operating frequency analysis based on operation distance revealed that micro-switch usage level has been categorized by 6 level after grouping similar Micro-switch based on movement data in one round trip from line 7's operation recording system in 2 cars. Based on Metro Operator's management information system data, annual running distance, operation distance and number of stations have been factored into concluding number of annual round trips. As a result, Metro line A's annual average round trips has found to be 1,224 times.

\subsection{Micro-switch FMEA analysis}

FMEA(Failure Modes Effects and Criticality Analysis) sheet has been created based on day time controller Microswitch's BOM(Bill of Material) to see failure impact upon analysis. Assuming that micro-switch's Failure Detection Rate is the same, severity and occurrence frequency have been applied to analyze micro-switch's RPN(Risk Priority Number) value which causes serious failures.

Micro-switch's RPN value is followed by Table 4. P1 and P2 micro-switches had highest figure, 30. Compared to operating frequency, RPN value found to be relatively high. However, considering P5 and P6 micro-switches with the same occurrence rate that shows different RPN value by severity, it means that failures take place in field must take into account environmental factors such as contacts maintaining shape, contacts location at master controllers and operator's habits. Railway operator should not only presenting RPN value but also prepare plans to present RPN value to a competent level.

\subsection{Micro-switch life analysis results}

Operating rate which is presented by manufacturer as life has been analyzed by taking operation characteristics into account. Even if the parts are the same, usage level was different by working locations and purposes. Also if electrical life such as 1 million which is presented by manufacturer is taken into account, the life-span goes 2.4 408 years. Under these circumstances, preventive maintenance upon advent of life cycle becomes very much unrealistic.

After analyzing micro-switch's replacement characteristics, we learned that it is more efficient to set forth different level of maintenance standards in accordance with severity level as well as operation impact and the cost. In other words, level 5 which means higher level of service failure in terms of severity should be replaced in a batch and level 3 and 4 should be replaced step by step. And for the rest, existing maintenance measure remains as the same. 
Table 5. Micro-switch RPN(Risk Priority Number)

\begin{tabular}{|c|c|c|c|c|c|c|c|}
\hline Category & Micro-switch & Impact of the failure & Severity & Frequency & $\begin{array}{c}\text { Detection } \\
\text { rate }\end{array}$ & RPN & Rank \\
\hline \multirow{8}{*}{$\begin{array}{l}\text { Direction } \\
\text { Handle }\end{array}$} & D1 micro-switch & B7 P4 recognition. TC and CC1 failure in recognition & 3 & 2 & 1 & 6 & 7 \\
\hline & D2 micro-switch & B7 P4 failure in recognition & 3 & 2 & 1 & 6 & 7 \\
\hline & D3 micro-switch & B7 B1 brake failure in recognition & 3 & 2 & 1 & 6 & 7 \\
\hline & D4 micro-switch & $\begin{array}{l}\text { B7 B1 brake failure in recognition } \\
\text { (emergency reverse) }\end{array}$ & 1 & 1 & 1 & 1 & 9 \\
\hline & D5 micro-switch & Neutral failure in recognition(TCMS) & 1 & 1 & 1 & 1 & 9 \\
\hline & D6 micro-switch & $\begin{array}{l}\text { Direction Controller Reverse } \\
\text { failure in recognition (ATC Card) }\end{array}$ & 1 & 1 & 1 & 1 & 9 \\
\hline & D7 micro-switch & $\begin{array}{l}\text { Direction Controller Neutral } \\
\text { failure in recognition (ATC Card) }\end{array}$ & 1 & 1 & 1 & 1 & 9 \\
\hline & D8 micro-switch & $\begin{array}{l}\text { when forward and Reverse, } \\
\text { Failure in recognition of Battery Contactor }\end{array}$ & 1 & 2 & 1 & 2 & 9 \\
\hline \multirow{9}{*}{ Reverse handle } & P1 micro-switch & $\begin{array}{c}\text { Direction Controller Forward } \\
\text { failure in recognition (TC Card) }\end{array}$ & 5 & 6 & 1 & 30 & 1 \\
\hline & P2 micro-switch & $\begin{array}{l}\text { Direction Controller Forward failure in recognition } \\
\text { (CC1 Card) }\end{array}$ & 5 & 6 & 1 & 30 & 1 \\
\hline & P3 micro-switch & $\begin{array}{c}\text { Direction Controller Forward } \\
\text { failure in recognition (ATC Card) }\end{array}$ & 4 & 4 & 1 & 16 & 3 \\
\hline & P4 micro-switch & $\begin{array}{l}\text { Direction Controller Reverse } \\
\text { failure in recognition (TC Card) }\end{array}$ & 4 & 4 & 1 & 16 & 3 \\
\hline & P5 micro-switch & $\begin{array}{l}\text { Direction Controller Reverse } \\
\text { failure in recognition (CC1 Card) }\end{array}$ & 4 & 5 & 1 & 20 & 2 \\
\hline & P6 micro-switch & Neutral failure in recognition(ATC) & 2 & 5 & 1 & 10 & 6 \\
\hline & P7 micro-switch & Full Service Brake failure in recognition(ATC) & 2 & 1 & 1 & 2 & 8 \\
\hline & P8 micro-switch & $\mathrm{P} 0.5 \sim \mathrm{P} 4$ reverse failure in recognition(TCMS) & 3 & 5 & 1 & 15 & 4 \\
\hline & P9 micro-switch & $\begin{array}{c}\text { P0.5 P4 reverse failure in recognition } \\
\text { (emergency reverse) }\end{array}$ & 3 & 5 & 1 & 15 & 4 \\
\hline \multirow{2}{*}{$\begin{array}{l}\text { Dead man } \\
\text { handle }\end{array}$} & DMS1 & Dead Man switch failure in recognition & 4 & 3 & 1 & 12 & 5 \\
\hline & DMS2 & Dead Man switch failure in recognition & 4 & 3 & 1 & 12 & 5 \\
\hline
\end{tabular}

\section{Prediction by using Field Data}

\subsection{Collection data}

Total numbers of micro-switch installed in the line A was 966. To analyze failure rate of the field data, we collected data of 7 years. Data is extracted from metro's management information system. To comply with operator's data protection policy, the data has been processed.

To learn failure distribution of collected micro-switch data, we ran a Weibull analysis for pre collected Microswitch. One of merits of Weibull analysis is that it captures beginning of bathtub curves, normal condition and abrasion.

By analyzing failure data, prediction of future failure becomes possible. micro-switch's field data shown in
Table 7 refers to consolidated data of Micro-switch related to the origin vehicle failures and data of batch replacements for preventive maintenance purpose.

\subsection{Data analysis}

We used RELEX's Weibull module, a reliability analysis program to analyze Table 6's P1 micro-switch field data.

Relay's Weibull analysis showed that shape parameter ( $\beta$ ) was 1.0678, scale parameter( $\eta$ ) was 28,418.2734.

Regards to average life, according to Weibull contribution, average life is as below.

$$
E(t)=M T B F=\eta \times \Gamma\left(1+\frac{1}{m}\right)
$$


Table 6. Micro-switch life analysis results

\begin{tabular}{|c|c|c|c|c|c|c|}
\hline Usage level & Micro-switch & $\begin{array}{l}\text { Operating freq. per } \\
\text { round trip }\end{array}$ & Electrical life & Annual round trips & $\begin{array}{c}\text { Annual operating } \\
\text { times }\end{array}$ & life span \\
\hline \multirow{2}{*}{6} & P1 & 332 & \multirow{18}{*}{1 Mil. times } & \multirow{18}{*}{1,224 times } & 406,368 times & 2.4 \\
\hline & $\mathrm{P} 2$ & 332 & & & 406,368 times & 2.4 \\
\hline \multirow{4}{*}{5} & P8 & 176 & & & 215,424 times & 4.6 \\
\hline & P9 & 176 & & & 215,424 times & 4.6 \\
\hline & P5 & 175 & & & 214,200 times & 4.6 \\
\hline & P6 & 175 & & & 214,200 times & 4.6 \\
\hline \multirow{2}{*}{4} & P3 & 156 & & & 190,944 times & 5.2 \\
\hline & $\mathrm{P} 4$ & 156 & & & 190,944 times & 5.2 \\
\hline 3 & DMS & 146 & & & 178,704 times & 5.6 \\
\hline \multirow{4}{*}{2} & D1 & 2 & & & 2,448 times & 408 \\
\hline & D2 & 2 & & & 2,448 times & 408 \\
\hline & D3 & 2 & & & 2,448 times & 408 \\
\hline & D8 & 2 & & & 2,448 times & 408 \\
\hline \multirow{5}{*}{1} & D4 & 0 & & & & - \\
\hline & D5 & 0 & & & & - \\
\hline & D6 & 0 & & & & - \\
\hline & D7 & 0 & & & & - \\
\hline & $\mathrm{P} 7$ & 0 & & & & - \\
\hline
\end{tabular}

$$
\begin{aligned}
& =28418.2734 \times \Gamma\left(1+\frac{1}{1.0678}\right) \\
& =28418.2734 \times \Gamma(1.936505) \\
& =28418.2734 \times 0.974796 \\
& =27702.02 \text { hours }
\end{aligned}
$$

$($ where, $\mathrm{m}=\beta, \Gamma(1.936505)=0.974796)$

According to gamma function, average life (MTTF) is approximately 27,702 hours.

Also shape parameter $(\beta)$ is 1.0678 which shows Microswitch approximates distribution value and it also had distribution with characteristics of electronics. To summarize, failures are not random occurrence, thus it does not conforms trend in failures.

Considering the fact that most railway vehicles' parts of railway operator have no life data, meaningful field data should be secured by the operator through long period of maintenance experience to get reliable life data analysis. If not secured, the operator can refer to prediction standards and manufacturer standards for the purpose of comparing field data collected from maintenance experience. By doing this, the operator can put forward best suited parts life for the railway operator.
Refer Fig. 1 and Fig. 2 for probability distribution and reliability distribution

\section{Reliability prediction by using prediction standards}

\subsection{Reliability prediction of model parts}

Traditional model such as MIL-HDBK-217F defines failure prediction by multiplying basic failure rate by factors such as environment, quality, stress and temperature as shown in Table 8. Multiplying model's weakness is that prediction of failure rates is sensitive towards basic failure rate and other external factors. Also failure mechanism has not been fully considered.

$$
\lambda_{\mathrm{p}}=\lambda_{\mathrm{b}} \pi_{\mathrm{E}} \pi_{\mathrm{Q}} \pi_{\mathrm{S}} \pi_{\mathrm{T}}
$$

( $\lambda_{b}$ basic failure rate, $\pi_{E}$ : environment factor, $\pi_{Q}$ : Quality factor, $\pi_{S}$ : Stress factor, $\pi_{T}$ : Temperature factor)

Reliability information analysis center presented a complex model which combines multiplying and adding model to supplement the weakness and minimize the errors in prediction as shown in Formula 2

$$
\begin{aligned}
& \lambda_{\mathrm{p}}=\lambda_{\mathrm{o}} \pi_{\mathrm{o}}+\lambda_{\mathrm{e}} \pi_{\mathrm{e}}+\lambda_{\mathrm{c}} \pi_{\mathrm{c}}+\lambda_{\mathrm{i}}+\lambda_{\mathrm{sj}} \pi_{\mathrm{sj}} \\
& \lambda_{\mathrm{p}} \text { : Prediction failure rate }
\end{aligned}
$$


Table 7. Number A Line's micro-switch replacement status

\begin{tabular}{|c|c|c|c|c|c|c|c|}
\hline & \multicolumn{7}{|c|}{ Number A Line's micro-switch replacement status } \\
\hline & 2005 & 2006 & 2007 & 2008 & 2009 & 2010 & 2011 \\
\hline D1 & 2 & 2 & 3 & 0 & 3 & 2 & 0 \\
\hline D2 & 1 & 0 & 2 & 0 & 6 & 1 & 0 \\
\hline D3 & 3 & 6 & 2 & 1 & 3 & 2 & 0 \\
\hline D4 & 0 & 0 & 2 & 2 & 3 & 2 & 1 \\
\hline D5 & 0 & 0 & 1 & 3 & 2 & 2 & 4 \\
\hline D6 & 0 & 1 & 3 & 2 & 4 & 2 & 1 \\
\hline D7 & 0 & 2 & 0 & 1 & 2 & 0 & 3 \\
\hline D8 & 1 & 2 & 2 & 1 & 0 & 0 & 3 \\
\hline D9 & 0 & 0 & 2 & 2 & 2 & 1 & 0 \\
\hline D10 & 2 & 3 & 5 & 2 & 2 & 0 & 1 \\
\hline D11 & 2 & 3 & 1 & 2 & 2 & 2 & 0 \\
\hline D12 & 0 & 0 & 0 & 1 & 3 & 0 & 0 \\
\hline D13 & 0 & 0 & 0 & 1 & 4 & 1 & 0 \\
\hline P1 & 5 & 2 & 2 & 5 & 4 & 1 & 1 \\
\hline $\mathrm{P} 2$ & 5 & 2 & 1 & 5 & 2 & 3 & 2 \\
\hline P3 & 0 & 4 & 3 & 6 & 7 & 1 & 0 \\
\hline P4 & 0 & 7 & 2 & 8 & 5 & 2 & 1 \\
\hline P5 & 0 & 0 & 1 & 4 & 1 & 0 & 0 \\
\hline P6 & 0 & 1 & 0 & 5 & 0 & 1 & 0 \\
\hline P7 & 0 & 1 & 1 & 4 & 0 & 0 & 2 \\
\hline P8 & 0 & 0 & 1 & 7 & 4 & 0 & 2 \\
\hline P9 & 0 & 3 & 3 & 2 & 0 & 0 & 1 \\
\hline P10 & 0 & 0 & 0 & 1 & 0 & 0 & 0 \\
\hline P11 & 0 & 0 & 0 & 0 & 1 & 0 & 1 \\
\hline P12 & 0 & 0 & 1 & 0 & 1 & 0 & 1 \\
\hline DMS1 & 1 & 3 & 4 & 11 & 2 & 1 & 3 \\
\hline DMS2 & 1 & 3 & 4 & 11 & 2 & 1 & 4 \\
\hline Total & 23 & 45 & 46 & 87 & 65 & 25 & 31 \\
\hline
\end{tabular}

$\lambda_{\mathrm{o}}$ : Movement stress failure rate

$\pi_{\mathrm{o}}$ : Double multiplier by failure rate from movement stress

$\lambda_{\mathrm{e}}$ : Environment stress failure rate

$\pi$ : Double multiplier by failure rate from environment stress

$\lambda_{\mathrm{c}}$ : Electricity and temperature cycling stress

$\pi_{\mathrm{c}}$ : Double multiplier by electricity and temperature cycling stress from failure rate

$\lambda$ : Failure rate from inducement stress

$\lambda_{\mathrm{sj}}$ : Failure rate of soldered contacts

$\pi_{\mathrm{sj}}$ : Double multiplier by failure rate from soldered contact stress
As can be seen in formula 3, prediction of failure rate provides different rates by operating, environment, cycling, inducement, and contacts. 217 Plus provides 12 types of parts and software model which is called RiAC Rate. Parts models are composed of capacitor, diode, integrated circuit, inductor, transformer, optoelectronics device, switch, relay, connector, resistance, thyristor, and transistor. By each model, table 4 is being modified and used.

As micro-switch, if parts do not fall under 12 models, the prediction can utilize failure rate library which was already set. EPRD, a handbook that contains various failure rates from electronics, electronic part for reliability prediction purpose or NPRD, a failure rate library for non- 
Jung-Geon Ji, Kun-Young Shin, Duk-Gyu Lee, Moon-Shuk Song and Hi Sung Lee / IJR, 5(1), 1-9, 2012

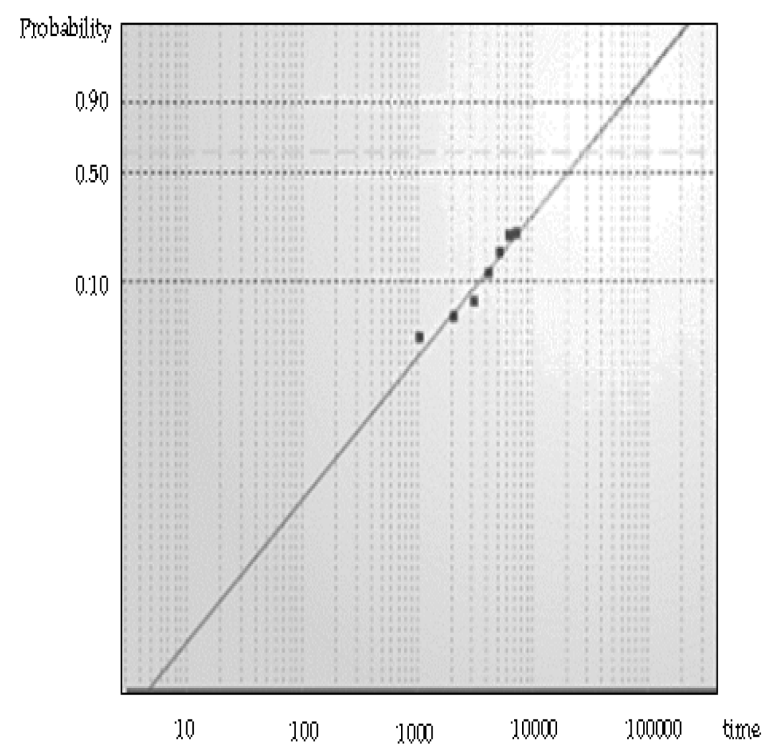

Fig. 1 P1 micro-switch probability distribution

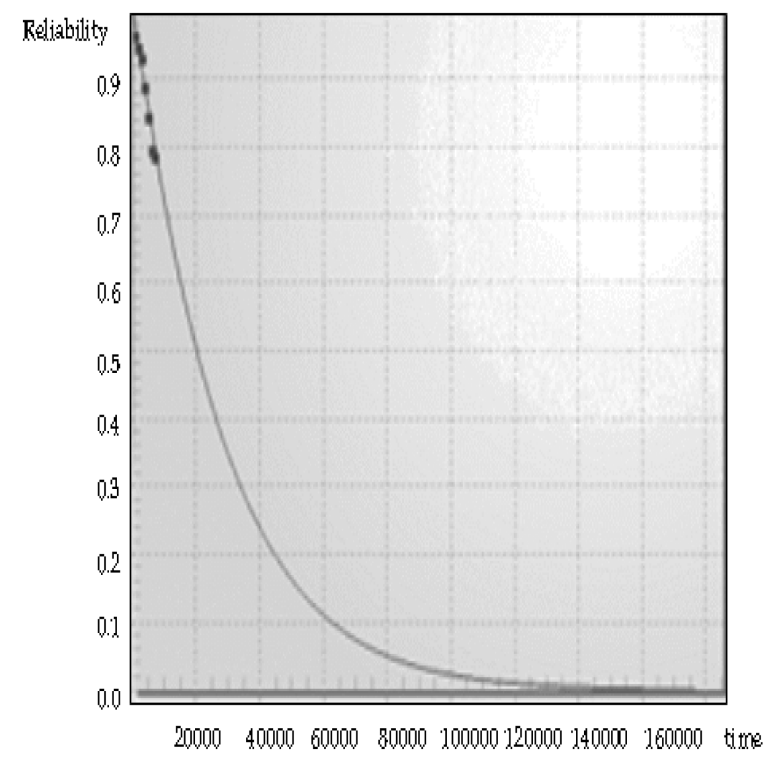

Fig. 2 P1 micro-switch reliability distribution

electronic parts can be used. This failure rate library has been developed to provide actual failure rate by parts unit which are divided by working condition and environment. Since it takes long time to build parts' mathematical failure rate model and technological development cycle becomes shorter than that of model building, not to mention growing complexity of parts over times. So microswitch's reliability prediction adopted EPRD/NPRD while using RELEX 2009, a reliability analysis program.

Failure rate library is from NPRD and life distribution is
Table 8. Reliability prediction results

\begin{tabular}{c|c}
\hline Value & Results \\
\hline Failure Rate, Predicted & 47.610000 \\
\hline MTTF, Predicted & 21004 \\
\hline Reliability, Predicted & 0.995250 \\
\hline Availability & 1.000000 \\
\hline MTTR & 0.000000 \\
\hline
\end{tabular}

value distribution. It is assumed that each part follows series configuration. Temperature at operating condition was $40^{\circ} \mathrm{C}$ and environment condition met $\mathrm{GM}$ (Ground Mobile). P1 micro-switch's average life was 21,004 hours, failure rate was 47.61 .

\section{Conclusions}

To analyze Micro-switch's life which is installed in Metro line number A's master controller and to develop preventive maintenance standards, life predictions have been conducted by various prediction methods. Firstly, parts lifeoperating frequency as presented by manufacturer-has been predicted by taking vehicle operation characteristics, usage frequency and severity level on top of FMEA analysis. Secondly failure distribution characteristics and parts average life have been analyzed by taking field data collected from local field. Lastly, reliability program, NPRD standard, has been utilized to draw conclusions.

1. Based on manufacturer's life(operating frequency), usage frequency and severity standards have been newly provided by considering vehicle operation characteristics. Also based on data left in vehicle operation recording system, micro-switch's operating frequency had been calculated. As a results, by manufacturer's life(operating frequency) standards, P1, P2 Micro-switches fell under 2.4 years, P8 and P9 fell under 4.6 years category which consist of 6 category.

2. As shown in reliability prediction result, predicted life varied by prediction methods. First, P1 Micro-switch's average life was approximately 2.39 years which drawn from NPRD that predicts system failure rate by adding parts' failure rate. When manufacturer standards were applied, P1 Micro-switch's average life was about 2.4 years. And field data's Weibull analysis showed P1 microswitch's average life as about 3.17 years.

3. After analyzing 21 micro-switches that are working under the same operation environment as number 7 line, we were able to come up with RPN value through FMEA analysis. The value refers that P1, P2 Micro-switch as $\mathrm{RPN}=30$, P5 Micro-switch as RPN=20, P3 Micro-switch 
as $\mathrm{RPN}=16$. The value can be as priority guideline upon vehicle maintenance, management and adjustment.

From this study, we learned that the life of railway vehicle parts can be obtained by referring to field data, standards for prediction, and manufacturer standards. Field data analysis showed that P1 micro-switch would have 12 times of life cycle if the maximum life of the railway vehicle is 40 years. FMEA analysis also showed that the change of the micro-switch of the master controller is inevitable. Thus to prevent a critical event such as a suspension of the vehicle operation, setting up the life cycle of micro-switch for a regular maintenance standard and its implementation is highly required. Though line A's P1 micro-switch's operating frequency is $1,015,920$ for major maintenance test period( 2.5 years or $250000 \mathrm{~km})$. Rather than referring to manufacture standards, it is recommended that the average life of P1 micro-switch obtained by a Weibull analysis of the field data should be applied in the non-regular maintenance standard as a special item to provide an efficient and reliable preventive maintenance standard to operators.

\section{References}

1. Shin, K. Y., Ji, J. G., Han, J. H, Lee, D. G., Son, Y. J. and Lee, H. S. (2011). "Life analysis and reliability prediction of relays based on life prediction method," Spring Conference of Korean Society for Railway.

2. RIAC-HDBK-217Plus (2006). "Handbook of 217PlusTM reliability prediction models," RIAC.

3. Jeon, T. B. (2006). "A reliability model of electronic ballasts for fluorescent lamp," Journal of the Korean Society for Quality Management, Vol. 35. No. pp.27-36

4. Lee, D. G. and Shim, J. H. (2010), "Reliability prediction using telcordia SR-332 Issue 2," Spring Conference of Korean Society for Railway.

5. Jang, J. S. (2004), "Reliability prediction guide," Kyo woo sa.

6. Jeon, T. B. (2010), "Reliability prediction of electricity and electronics system," Kyo woo sa.

Received(March 6, 2012), Revised(March 9, 2012),

Accepted(March 12, 2012) 